\title{
Metaphyseal Burst Sign; the First Case Report of Subchondral Insufficiency Fracture of the Knee in Iran
}

\author{
Reza Noktehsanj ${ }^{1,2}$, Hamid-Reza Aslani ${ }^{3}$, Seyyed-Mohsen Hosseininejad ${ }^{4,5,{ }^{*}}$ and Mohammad-Amin Aslani ${ }^{6}$ \\ ${ }^{1}$ Department of Orthopedics, Fatemi Hospital, Ardabil University of Medical Sciences, Ardabil, Iran \\ 2 Bone, Joint, and Related Tissues Research Center, Akhtar Hospital, Shahid Beheshti University of Medical Sciences, Tehran, Iran \\ 3 Sport Medicine and Knee Research Center, Milad Hospital, Tehran, Iran \\ ${ }^{4}$ School of Medicine, Shahid Beheshti University of Medical Sciences, Tehran, Iran \\ ${ }^{5}$ Joint, Bone, and Connective Tissue Rheumatology Research Center, Golestan University of Medical Sciences, Gorgan, Iran \\ ${ }^{6}$ Tehran University of Medical Sciences, Tehran, Iran
}

* Corresponding author: Seyyed-Mohsen Hosseininejad, Joint, Bone, and Connective Tissue Rheumatology Research Center, Golestan University of Medical Sciences, Gorgan, Iran. Email: Hosseininejad.s.mohsen@gmail.com

Received 2020 August 21; Revised 2020 October 04; Accepted 2020 October 19.

\begin{abstract}
Introduction: Metaphyseal burst sign, which is soft tissue edema in the metaepiphyseal region, is defined as a novel initial sign of subchondral insufficiency fracture of the knee (SIFK).

Case Presentation: We reported the first Iranian case of the SIFK in femoral medial condyle characterized by a metaphyseal burst sign which could be an early sign of the SIFK. Early diagnosis and treatment could dramatically relieve the sign and symptoms of patients with significant improvement of the MRI scan.

Conclusion: The metaphyseal burst sign as the initial specific sign of SIFK could be helpful for the early diagnose and management of the osteonecrosis of the affected area. The SIFK could potentially progress into severe joints degenerative changes in a short time; therefore, it seems that an early diagnosis and treatment thereafter could help resolve the clinicoradiological signs and symptoms of SIFK. This could postpone knee osteoarthritis and the need for joint arthroplasty.

Keywords: Magnetic resonance imaging (MRI), Medial femoral condyle, Metaphyseal burst sign, Osteonecrosis, Subchondral insufficiency fracture
\end{abstract}

\section{Introduction}

Subchondral insufficiency fracture of the knee (SIFK), which has replaced the entity of spontaneous osteonecrosis of the knee (SONK) in the latest literature $(1,2)$, is characterized as severe knee pain in the absence of trauma or following a minor trauma usually in people above 65 years of age $(3,4)$. The most common location of SIFK is in the medial femoral condyle; however, it has been reported in the lateral femoral condyle and the tibial condyles as well $(5,6)$.

Exact etiology of the SIFK is still a matter of debate; nevertheless, the current popular etiology is that these insufficiency fractures result in the accumulation of fluid in the bone marrow, leading to focal ischemia and subsequent bony necrosis $(3,7$, 8). It is important to keep in mind that this condition can potentially lead to the rapid deterioration of osteoarthritis and functional disabilities (9).

The clinicoradiological classification system was expressed in four stages by Soucacos in 1997. The first stage is characterized by bone marrow edema alone while the second includes progression to initial flattening of the femoral condyle. These first two stages are diagnosed by magnetic resonance imaging (MRI) as significant changes are not detectable on plain radiographs. The third and fourth stages are classified as the 'crescent sign' and the collapse of the subchondral bone, respectively (8-12).

The early diagnosis in the first two stages is important as conservative therapy is usually effective 11,13 . The most frequently used diagnostic criteria on MRI are an area of bone marrow edema in affected condyle associated with subchondral bone changes $(4,5,9,13-19)$. Treatment of SIFK in the initial stages consists of protected weight-bearing, non-steroidal anti-inflammatory medications (NSAIDs), analgesic agents, and bisphosphonates (17, 20). The metaphyseal burst sign, edema, is defined as an increased signal on fluid sensitive sequences that involve the soft tissues overlying the epimetaphyseal cortex of the affected condyle (21).

The present study aimed to present the first case of the SIFK in Iran with an additional early MRI sign of SIFK (i.e., the metaphyseal burst sign) which can be helpful for an earlier diagnosis.

\section{Case Presentation}

The case is a 45-year-old otherwise healthy male medical doctor with a complaint of pain in the right knee. He described a moderate pain in the medial aspect of the knee which became worse with weight bearing. He also complained of pain at rest; however, there was no report of knee locking and giving way.

Copyright (c) 2020, Author(s). This is an open-access article distributed under the terms of the Creative Commons Attribution-NonCommercial 4.0 International License (http://creativecommons.org/licenses/by-nc/4.0/) which permits copy and redistribute the material just in noncommercial usages, provided the original work is properly cited 

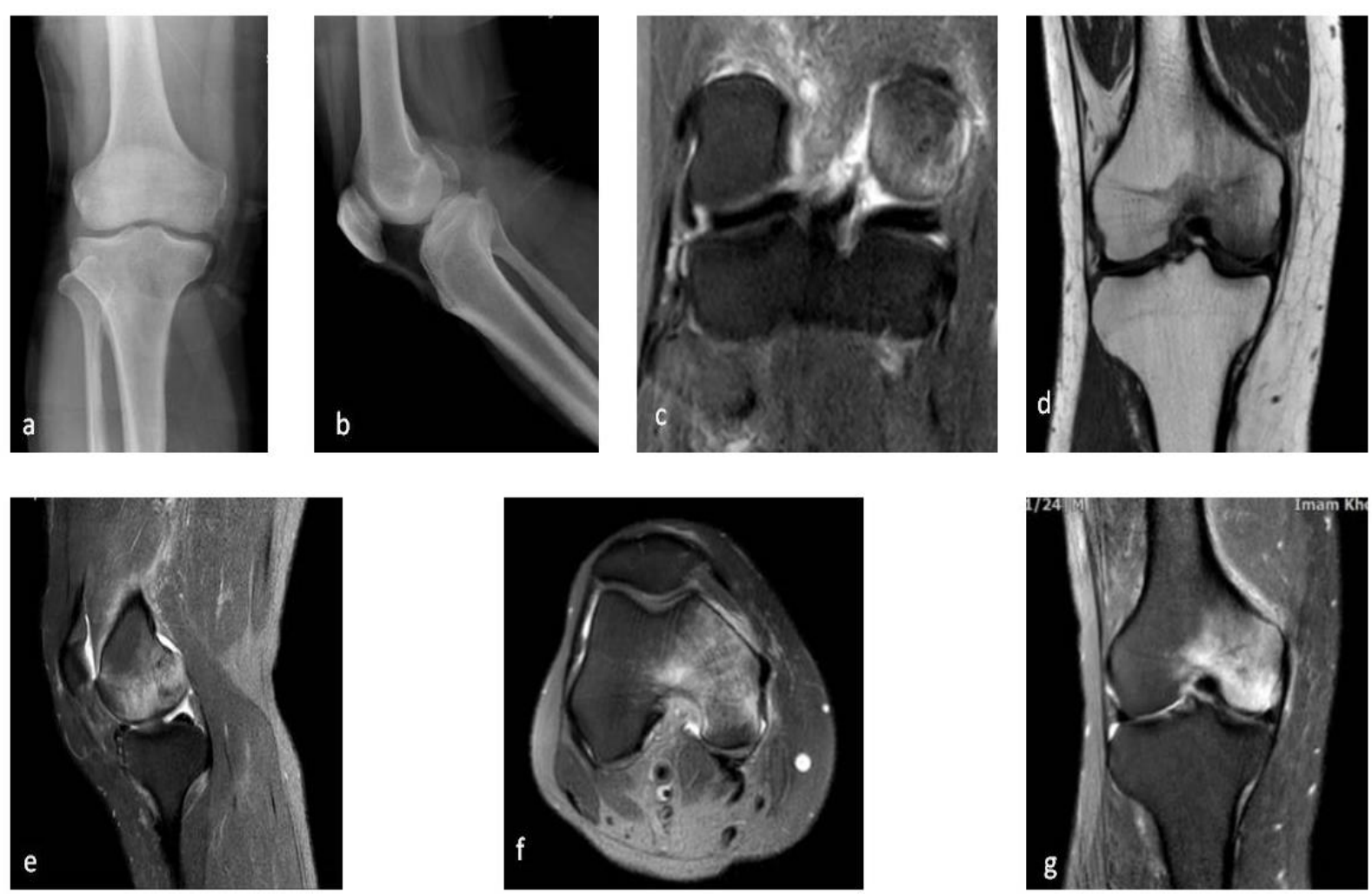

Figure 1. Initial plain radiography and MRI evaluation of the right knee anteroposterior (a), lateral (b), and X-rays showed no significant abnormality. Coronal T2 (c) depicted intact posterior horns of the meniscus, and coronal T1 (d), sagittal T2 (e), and axial T2 (f) illustrated edematous change involving the medial femoral condyle. Coronal STIR (g) portrayed the typical pattern of bone marrow edema and metaphyseal burst sign

His body mass index was $32 \mathrm{~kg} / \mathrm{m}^{2}$ and he had no history of previous trauma, knee surgery, coagulopathy, or steroids reception. He was an exsmoker and consumed alcohol occasionally. On Physical examination, he had medial femoral condyle point tenderness. Mild knee effusion was detected as well. The range of motion of the knee was not remarkable; nevertheless, full knee flexion was painful. McMurray, pivot shift, and varus/valgus stress tests were normal.

Plain radiographs of the affected knee were normal (Figure 1). MRI was requested which showed extensive edema in the medial femoral condyle extended to the intercondylar notch and the lateral condyle besides metaphyseal subperiosteal edema of the distal femur "metaphyseal burst sign" (Figure 2). No malalignment was noticed in the lower limb X-ray alignment view.

The patient was ordered to decrease physical activities with protected weight bearing by crutches and take Celexib $100 \mathrm{mg}$ capsule twice a day plus alendronate (Osteofos) $70 \mathrm{mg}$ tablet weekly for 6 weeks. On the next follow-up visit after 6 weeks, his signs and symptoms were relieved, his knee XRay was normal, and the MRI illustrated a significant decrease of the edema of the distal femur (Figure 2).

The NSAID was discontinued while he was suggested to continue taking alendronate as before. He
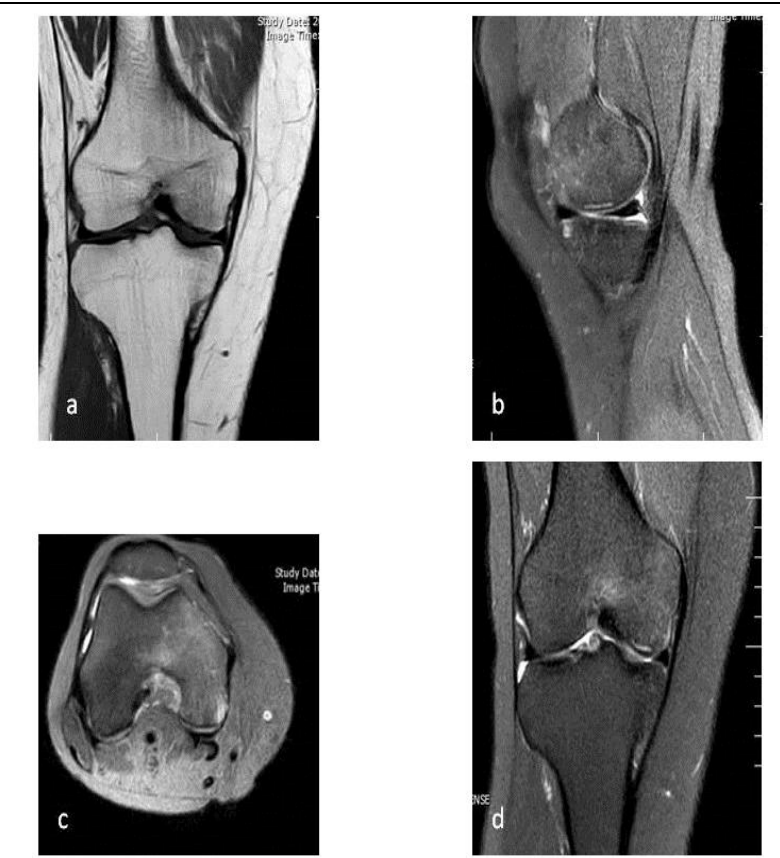

Figure 2. Second MRI which was taken 6 weeks after conservative treatment, including non-weight bearing, nonsteroidal anti-inflammatory medications, and bisphosphonates. Coronal T1(a), sagittal T2 (b), axial T2 (c), and coronal STIR (d) weighted images show a decrease in the bone marrow edema, compared to before. $b$ and d illustrate the natural appearance of the focal linear subchondral hyperintensity in femoral medial condyle 

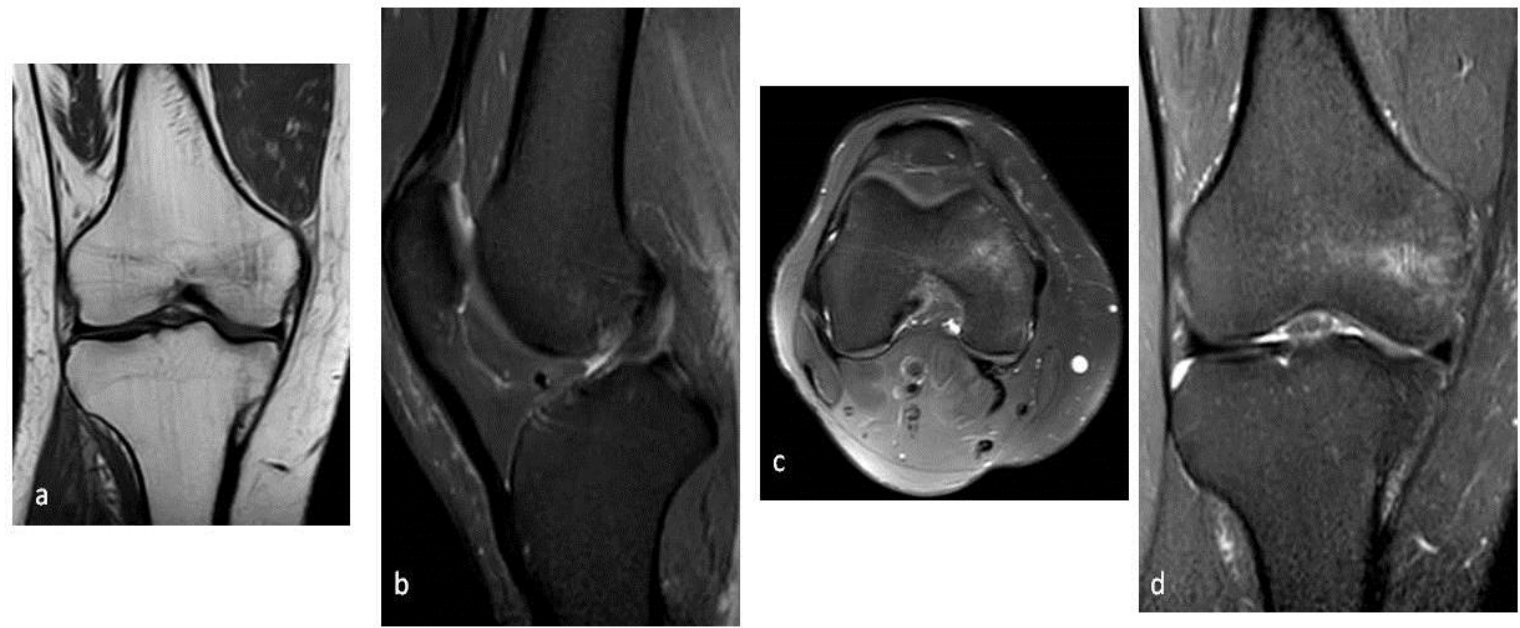

Figure 3. Coronal T1 (a), sagittal STIR (b), axial T2 (c), and coronal STIR (d) weighted images depict the significant decrease of medial condyle of femur bone marrow edema and subsidence of focal subchondral linear hyperintensity

continued to feel better as the pain significantly decreased and was able to walk without crutches. The second follow-up MRI after 3 months showed significant shrinkage of the edematous area (Figure 3).

\section{Discussion}

Extensive bone marrow edema extending from the subchondral bone plate into the epiphyseal region with variable involvement of the metaphysis on MRI without a traumatic event is suggestive of SIFK (22). However, it was not found in other conditions, such as a tumor, infection, acute osteochondral fracture, and osteoarthritis (21).

We reported the 'metaphyseal burst sign' (Figure 1) as an early sign of SIFK in a 45-year-old Iranian male. To the best of our knowledge, this is the first reported case in this regard in Iran. In this case, metaphyseal burst sign was present in combination with the unicondylar bone marrow edema. On MRI, it was recognized by edema of the tissues overlying the metaphyseal region of the affected condyle where the nutrient vessels perforate the bone to supply blood to the epimetaphysis and subchondral bone (23-25). These could be indicative of the vascular origin of the metaphyseal burst sign. They are related to the sudden increase of intraosseous pressure consequent to a subchondral insufficiency fracture. This fracture could cause the loss of the integrity of the subchondral bone plate leading to a retrograde raise of arterial and venous blood pressure with resultant extravasations.

There would be several differential diagnoses including previous traumatic injury to the knee, and/or meniscus, especially root avulsion, and ligament injuries which were not relevant to our case due to normal knee stability tests and negative history for recent trauma. Besides, the proximal tibia of the patient was not remarkable based on examination and MRI $(21,26)$. Treatment of such lesion consists of activity modification, NSAIDs, bisphosphonate, and regular follow-up visits. The definite treatment in case of severe degenerative changes would be joint arthroplasty (17).

In conclusion, the metaphyseal burst sign as the initial specific sign of SIFK could be helpful for the early diagnose and management of the osteonecrosis of the affected area. The SIFK could potentially progress into severe joints degenerative changes in a short time; therefore, it seems that an early diagnosis and treatment thereafter could help resolve the clinicoradiological signs and symptoms of SIFK. This could postpone knee osteoarthritis and the need for joint arthroplasty.

\section{Acknowledgements}

The authors would like to give their special thanks to the patient for his cooperation.

\section{Footnotes}

Conflict of Interests: The authors declare that there was no conflict of interest in this study.

Ethical considerations: Written informed consent was obtained from the patient and the study was conducted based on the Declaration of Helsinki.

\section{References}

1. Atoun E, Segal G, Debi R, Lubovsky O, Djabbarov R, Peskin B, Falah M, Mor A, Elbaz A. Gait assessment of patients with spontaneous osteonecrosis of the knee. OsteoarthrCartil. 2016;24(S394).

2. Gorbachova T, Melenevsky Y, Cohen M, Cerniglia BW. Osteochondral lesions of the knee: differentiating the most common entities at MRI. Radiographics. 2018; 38(5):14781495. doi: 10.1148/rg.2018180044. [PubMed: 30118392].

3. Yamamoto T, Bullough PG. Spontaneous osteonecrosis of the knee: the result of subchondral insufficiency fracture. JBJS. 
2000;82(6):858-66. doi: 10.2106/00004623-20000600000013. [PubMed: 10859106].

4. Yamamoto T, Bullough PG. Subchondral insufficiency fracture of the femoral head and medial femoral condyle. SkeletRadiol. 2000; 29(1):40-4. doi: 10.1007/s002560050007. [PubMed: 10663588].

5. Narvaez JA, Narvaez J, De Lama E, Sanchez A. Spontaneous osteonecrosis of the knee associated with tibial plateau and femoral condyle insufficiency stress fracture. Eur Radiol. 2003;13(8): 1843-8. doi: 10.1007/s00330-002-1775-6. [PubMed: 12942284].

6. Lotke PA, Nelson CL, Lonner JH. Spontaneous osteonecrosis of the knee: Tibial plateaus. Orthop Clin North Am. 2004; 35(3):365-70. doi: 10.1016/j.ocl.2004.02.009. [PubMed: 15271545].

7. Soucacos PN, Xenakis TH, Beris AE, Soucacos PK, Georgoulis A. Idiopathic osteonecrosis of the medial femoral condyle. Classification and treatment. Clin Orthop Relat Res. 1997; (341):82-9. [PubMed: 9269159].

8. Pape D, Seil R, Kohn D, Schneider G. Imaging of early stages of osteonecrosis of the knee. Orthop Clin North Am. 2004; 35(3):293-303. doi: 10.1016/j.ocl.2004.04.001. [PubMed: 15271537].

9. Sokoloff RM, Farooki S, Resnick D. Spontaneous osteonecrosis of the knee associated with ipsilateral tibial plateau stress fracture: report of two patients and review of the literature. Skeletal Radiol. 2001;30(1):53-6. doi: 10.1007/s002560000290. [PubMed: 11289636].

10. Hussain ZB, Chahla J, Mandelbaum BR, Gomoll AH, Laprade RF. The role of meniscal tears in spontaneous osteonecrosis of the knee: a systematic review of suspected etiology and a call to revisit nomenclature. Am J Sports Med. 2019;47(2):501-507. doi: 10.1177/0363546517743734. [PubMed: 29253348].

11. Sibilska A, Góralczyk A, Hermanowicz K, Malinowski K. Spontaneous osteonecrosis of the knee: what do we know so far? A literature review. Int Orthop. 2020;44(6)1063-69. doi: 10.1007/s00264-020-04536-7. [PubMed: 32249354].

12. Yates PJ, Calder JD, Stranks GJ, Conn KS, Peppercorn D, Thomas NP. Early MRI diagnosis and non-surgical management of spontaneous osteonecrosis of the knee. Knee. 2007;14(2):1126. doi: 10.1016/j.knee.2006.10.012. [PubMed: 17161606].

13. Karachalios T, Zibis A, Papanagiotou P, Karantanas AH, Malizos $\mathrm{KN}$, Roidis N. MR imagingfindings in early osteoarthritis of the knee. Eur J Radiol. 2017;50(3):225-30. doi:10.1016/j.ejrad. 2004.01.018.

14. Griffiths H. Spontaneous osteonecrosis. Orthopedics. 1986; 9(4):596, 598-602. [PubMed: 3960793].

15. Zywiel MG, McGrath MS, Seyler TM, Marker DR, Bonutii PM, MontMA. Osteonecrosis of the knee: a review of three disorders. Orthop Clin North Am. 2009;40(2):193-211. doi: 10.1016/j.ocl.2008.10.010. [PubMed: 19358905].
16. Moosikasuwan JB, Miller TT, Math K, Schultz E. Shifting bone marrow edema of the knee.Skeletal Radiol. 2004;33(7):380-5. doi:10.1007/s00256-004-0783-9. [PubMed: 15138729].

17. Karim AR, Cherian JJ, Jauregui JJ, Pierce T, Mont MA. Osteonecrosis of the knee: review. Ann Transl Med. 2015 3(1):6. doi: 10.3978/j.issn.2305-5839.2014.11.13. [PubMed: 25705638]

18. Lecouvet FE, van de Berg BC, Maldague BE, LebonCJ, Jamart J, Saleh $\mathrm{N}$ et al. Early irreversible osteonecrosis versus transient lesions of the femoral condyles: prognostic value of subchondral bone and marrow changes on MR imaging. AJR Am J Roentgenol. 1998; 170(1):71-7. doi: 10.2214/ajr.170.1.9423603. [PubMed: 9423603].

19. Barr MS, Anderson MW. The knee: bone marrow abnormalities. Radiol Clin North Am. 2017;40(5):1109-20. doi: 10.1016/s0033-8389(02)00051-9. [PubMed: 12462471].

20. Yang WM, Zhao CQ, Lu ZY, Yang WY, Lin DK, CaoXW. Clinical characteristics and treatment of spontaneous osteonecrosis of medial tibial plateau: a retrospective case study. Chin Med J (Engl). 2018;131(21):2544-50. doi: 10.4103/0366-6999.244113. [PubMed: 30381587].

21. Vidoni A, Shah R, Mak D, Beale D, Beale S, James $S$ et al. Metaphyseal burst sign: A secondary sign on MRI of subchondral insufficiency fracture of the knee. J Med Imaging Radiat Oncol. 2018; 62(6):764-8. doi: 10.1111/17549485.12781. [PubMed: 30113137].

22. Roemer FW, Frobell R, Hunter DJ, Crema MD, Fischer W, Bohndorf $\mathrm{K}$ et al. MRI-detected subchondral bone marrow signal alterations of the knee joint: terminology, imaging appearance, relevance and radiological differential diagnosis. Osteoarthritis Cartilage. 2009;17(9):1115-31. doi: 10.1016/j. joca.2009.03.012. [PubMed: 19358902].

23. Kirschner MH, Menck J, Hennerbichler A, Gaber O, Hofmann GO. Importance of arterial blood supply to the femur and tibia for transplantation of vascularized femoral diaphyses and knee joints. World J Surg. 1998;22(8):845-52. doi: 10.1007/ s002689900480. [PubMed: 9673557].

24. van der Woude JAD, van Heerwaarden RJ, Bleys RLAW. Periosteal vascularization of the distal femur in relation to distal femoral osteotomies: a cadaveric study. J Exp Orthop. 2016;3(1):6. doi: 10.1186/s40634-016-0042-8. [PubMed: 26915006].

25. Reddy AS, Frederick RW. Evaluation of the intraosseous and extraosseous blood supply to the distal femoral condyles. $\mathrm{Am} J$ Sports Med. 1998;26(3):415-9. doi: 10.1177/0363546598026 0031201. [PubMed: 9617405].

26. Bhatia S, LaPrade CM, Ellman MB, LaPrade RF. Meniscal root tears: significance, diagnosis, and treatment. Am J Sports Med. 2014;42(12):3016-30. doi: 10.1177/0363546514524162. [PubMed: 24623276]. 